\title{
Two cases of radiation-induced cutaneous angiosarcoma
}

\author{
Tomohiro Katagiri · Chikako Yamauchi • \\ Nami Ueki · Yasushi Nagata $\cdot$ Kiyotomo Matsugi • \\ Yukari Hattori · Eiji Takeuchi · Masahiro Hiraoka
}

Received: 11 August 2012/ Accepted: 9 November 2012/Published online: 20 December 2012

(C) The Japan Society of Clinical Oncology 2012

\begin{abstract}
We report two cases of radiation-induced cutaneous angiosarcoma that occurred years after radiotherapy for pelvic cancer. The tumors in both cases were inoperable because of the poor performance status of the patient or the extent of the lesion. We treated the tumors with concurrent chemotherapy and radiotherapy. The chemotherapy consisted of the weekly administration of $70 \mathrm{mg} / \mathrm{m}^{2}$ paclitaxel. Both patients received radiotherapy at the prescribed dose of 50 Gy in 2.0-Gy fractions. Two months after the treatment, the lesions of both cases diminished or disappeared. However, a few months after the treatment, both patients suffered from in-field local recurrence and distant metastases, and they subsequently died of their distant metastases. The initial responses of two cases to concurrent chemoradiotherapy were good, and
\end{abstract}

T. Katagiri · N. Ueki · M. Hiraoka

Department of Radiation Oncology and Image-Applied Therapy,

Graduate School of Medicine, Kyoto University, 54, Shogoin-

Kawahara-cho, Sakyo-ku, Kyoto 606-8507, Japan

e-mail: giriwo2@yahoo.co.jp

C. Yamauchi $(\bowtie) \cdot$ K. Matsugi

Department of Radiation Oncology, Shiga Medical Center

for Adults, Shiga, Japan

e-mail: chikay1796@gmail.com

\section{Y. Nagata}

Department of Radiation Oncology, Hiroshima University,

Hiroshima, Japan

Y. Hattori

Department of Dermatology, Shiga Medical Center for Adults,

Shiga, Japan

E. Takeuchi

Department of Pathology, Shiga Medical Center for Adults,

Shiga, Japan their quality of life was improved without severe complication. Although the long-term control of radiation-induced cutaneous angiosarcoma was difficult, chemoradiotherapy was one of the safe and effective palliative treatments.

Keywords Radiation-induced · Angiosarcoma · Chemoradiotherapy

\section{Introduction}

Angiosarcoma is rare, representing about $2 \%$ of soft tissue sarcoma and $5.4 \%$ of cutaneous soft tissue sarcoma [1]. Cutaneous angiosarcoma typically involves the head and neck, particularly the scalp and face. The etiology of angiosarcoma is poorly understood; however, several risk factors, such as radiation exposure and chronic lymph edema, have been reported [1]. The optimal treatment strategy for angiosarcoma is disputed. Surgery is often employed with or without radiotherapy for localized angiosarcoma, whereas radiotherapy is often used for unresectable cases and is usually avoided for radiationinduced angiosarcoma [1].

We report two cases of radiation-induced cutaneous angiosarcoma in the groin, which was treated with concurrent chemotherapy and radiotherapy.

\section{Case report}

Case 1

A 45-year-old woman with carcinoma of the endometrium underwent surgery (International Federation of Gynecology and Obsterics (FIGO) stage IB). She received adjuvant 


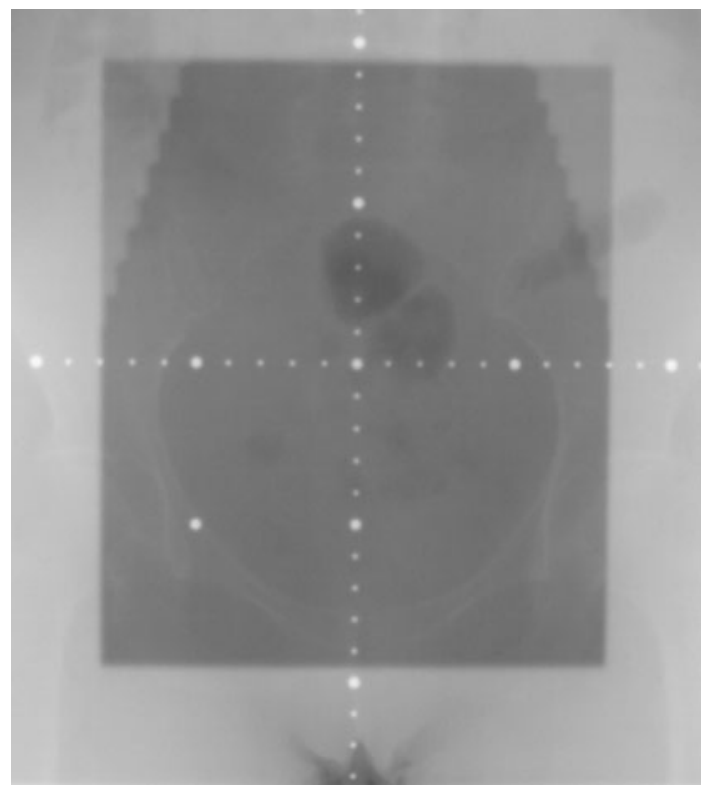

Fig. 1 A portal image taken during the adjuvant radiotherapy administered after surgery for carcinoma of the endometrium. The lower edge of the obturator foramen was used as the caudal border of the field

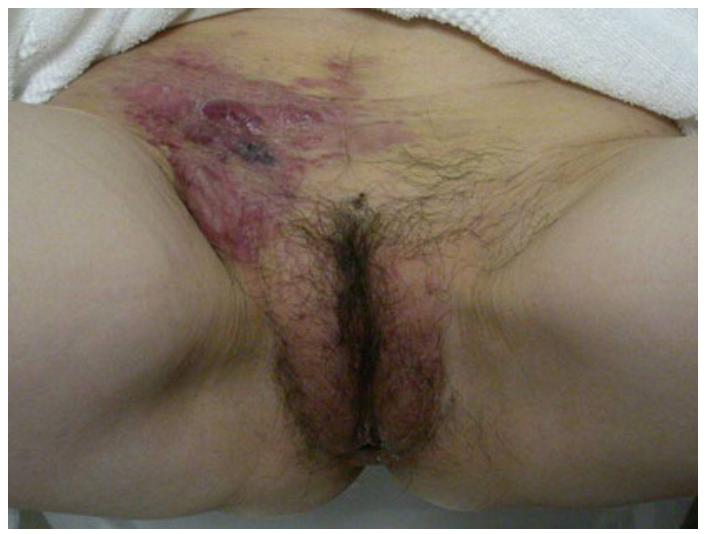

Fig. 2 A large reddish tumor extending from the right groin to the lower abdomen near the vulva

whole pelvic radiotherapy with high-energy X-rays (15 MV) consisting of 50.4 Gy (Fig. 1). A subsequent follow-up revealed no evidence of recurrence. However, 8 years after her initial treatment, she presented with a large reddish tumor extending from the right groin to the lower abdomen near the vulva (Fig. 2). There were no lymph node metastases or distant metastases. She underwent biopsy, which resulted in a diagnosis of angiosarcoma. The tumor was too large to be treated with curative surgery, so the treatment strategy was concurrent chemotherapy and radiotherapy. The tumor thickness was about $2.0 \mathrm{~cm}$ on the basis of the physical examination and CT examination, so $9-\mathrm{MeV}$ electron beam irradiation was

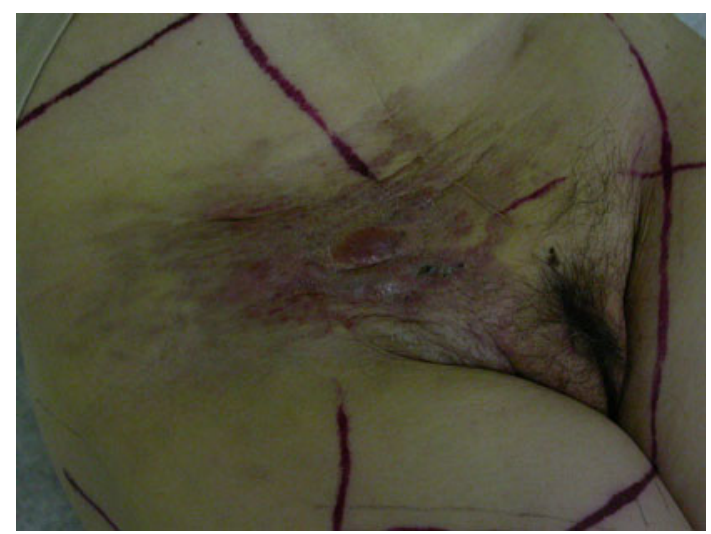

Fig. 3 The wide field $(26 \times 25 \mathrm{~cm}$, purple ink $)$ used for the electron beam irradiation

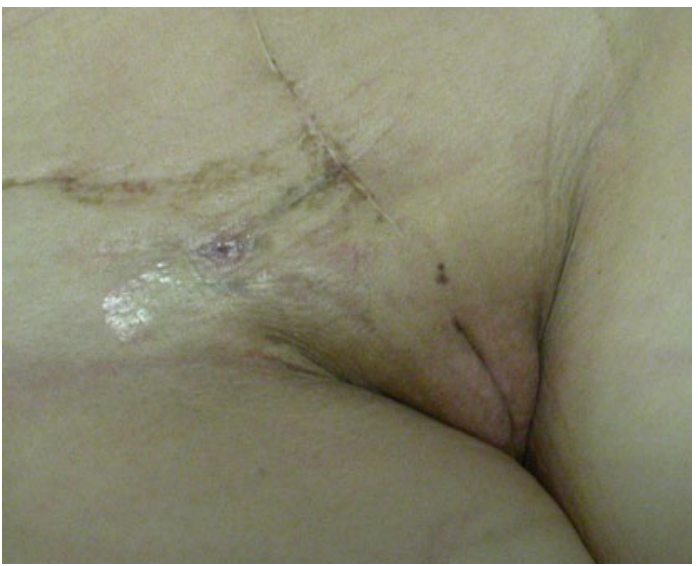

Fig. 4 Two months after the end of chemoradiotherapy, the tumor disappeared completely

delivered in a wide field $(26 \times 25 \mathrm{~cm}$, Fig. 3) with 2 Gy per fraction to a total dose of $50 \mathrm{~Gy}$, and a $0.5 \mathrm{~cm}$ bolus was used to ensure that the tumor was sufficiently irradiated. The concurrent chemotherapy consisted of the weekly administration of $70 \mathrm{mg} / \mathrm{m}^{2}$ paclitaxel (PTX) on days 1,8 , and 15 for 4 weeks. She received a total of 4 cycles. She completed the treatment, suffering only from grade 1 acute radiation dermatitis according to the National Cancer Institute Common Toxicity Criteria, version 4.0 (NCI-CTC ver.4). Adjuvant recombinant interleukin-2 (rIL-2) therapy was administered to her. The total administered dose was $2065 \times 10^{4}$ units. Two months after the end of the chemoradiotherapy, the tumor disappeared completely (Fig. 4). Two months later, a small nodule appeared near to the biopsy scar, and so a second biopsy was performed, which revealed the in-field local recurrence of the angiosarcoma. At the same time, distant metastases were detected in the lungs and the liver. Chemotherapy was administered again with weekly PTX, followed by epirubicin. However, she developed carcinomatous pleuritis and 
peritonitis and died 9 months after the start of the chemoradiotherapy.

\section{Case 2}

A 66-year-old woman with carcinoma of the cervix underwent preoperative chemotherapy and surgery (FIGO stage IIB). She received adjuvant whole pelvic radiotherapy consisting of 45 Gy. However, 15 years after her initial treatment, she presented with a mass in her right groin and the bleeding from the mass could not be controlled. She was admitted to our hospital. A physical examination revealed a bulky bleeding mass in the right groin and a small reddish lesion in the left groin (Fig. 5). There were no lymph node metastasis or distant metastasis. The lesion in her right groin was biopsied, which resulted in a diagnosis of angiosarcoma. Because of her poor performance status and old age, she could not undergo surgery. Thus, she was treated with concurrent chemotherapy and radiotherapy. The tumor thickness was about $4.0 \mathrm{~cm}$ on the basis of the physical examination and CT examination, so $12-\mathrm{MeV}$ electron beam irradiation was delivered in a wide field $(25 \times 25 \mathrm{~cm}, 1 \mathrm{~cm}$ bolus) with 2 Gy per fraction to a total dose of $36 \mathrm{~Gy}$. The concurrent chemotherapy consisted of the weekly administration of $70 \mathrm{mg} / \mathrm{m}^{2}$ PTX on days 1,8 , and 15 for 4 weeks. She received a total of 4 cycles. The tumor gradually decreased in size, and then 14 Gy was delivered in the shrunken fields with 6- to 9-MeV electron beams for each lesion on either side of her groin. The bleeding stopped as the radiotherapy proceeded, and she completed the treatment, suffering only from grade 2 acute radiation dermatitis according to NCI-CTC ver.4. She received adjuvant chemotherapy consisting of weekly PTX. However, 1 month after the end of the chemoradiotherapy, she developed distant metastases in the liver. Because of her poor performance status and clinical progression, she refused additional adjuvant therapy. Although the treated lesion was gradually shrinking without bleeding, the necrotic part persisted (Fig. 6), so this section was

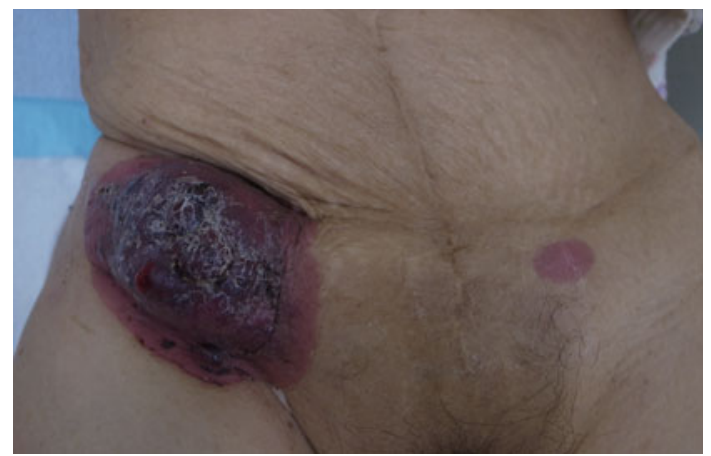

Fig. 5 A bulky bleeding tumor in the right groin and a small reddish tumor in the left groin

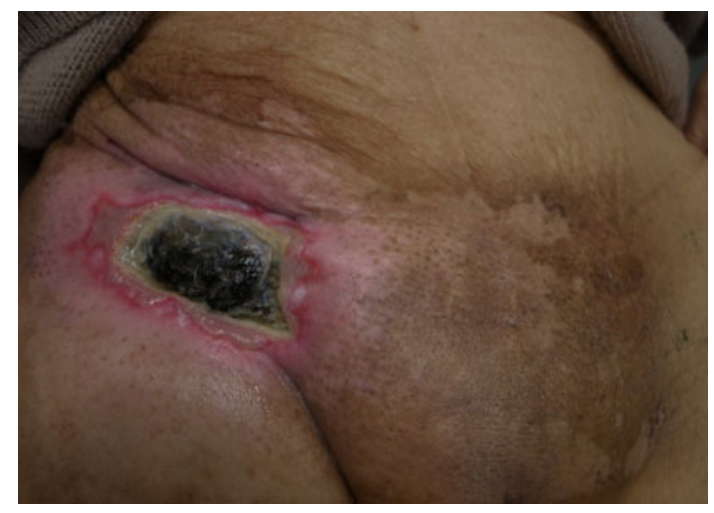

Fig. 6 One month after the end of the chemoradiotherapy, the tumor in the right groin was gradually shrinking without bleeding, and the tumor in the left groin disappeared completely

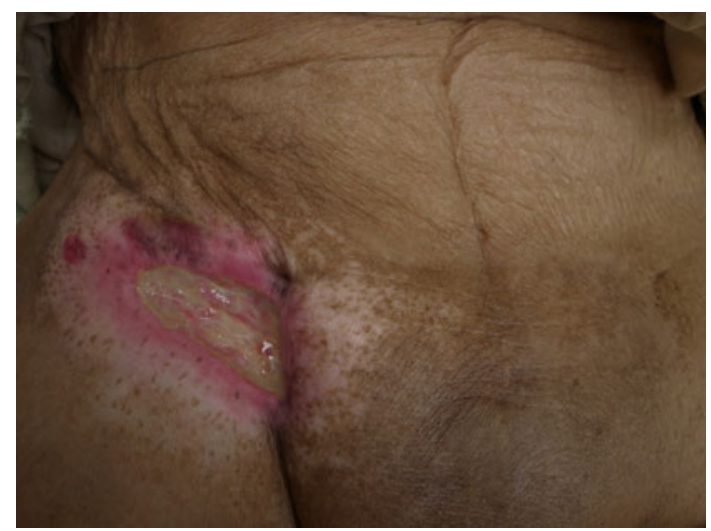

Fig. 7 Two months after the end of the chemoradiotherapy, the tumor was excised. A small erythema-like nodule appeared near the tumor and was suspected to be a recurrence

excised 1 month later, which resulted in ulceration (Fig. 7). A small erythema-like nodule appeared near the previous right groin mass within the radiation field 2 months after the end of the chemoradiotherapy. Distant metastases were also detected in the liver. Biopsy was not performed. Although the aforementioned nodule enlarged, the ulcerated part diminished and healed 1 month after the excision (Fig. 8). However, she developed carcinomatous pleuritis and died 5 months after the start of the chemoradiotherapy.

\section{Discussion}

Angiosarcoma is a rare malignant type of soft tissue sarcoma, which is often associated with radiotherapy. Most of the cutaneous angiosarcoma has been reported to arise in the scalp or the face. Cutaneous angiosarcoma rarely arises in the groin spontaneously. There have not been any randomized trials examining the optimal treatment strategy for angiosarcoma. Surgery is often performed for localized 


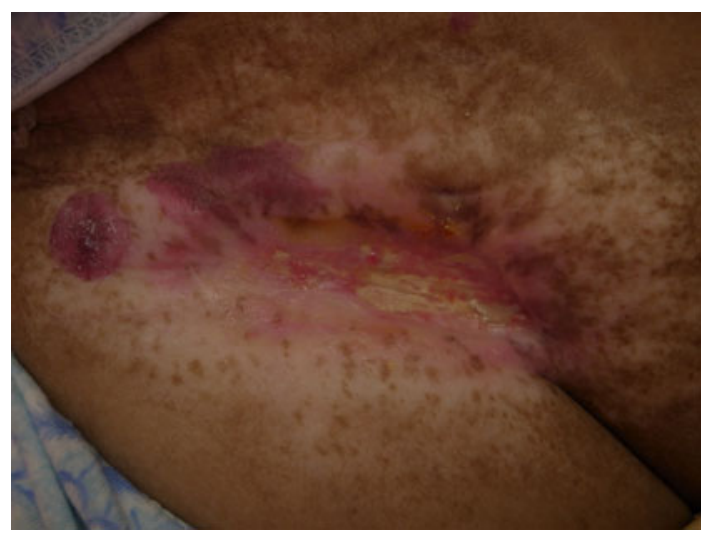

Fig. 8 One month after the excision, the ulcerated part, which resulted from the excision of the necrotic tissue, diminished and healed. The nodule, which was suspected to be a recurrence, was getting larger

angiosarcoma. In a single-institution report of angiosarcoma, the median survival time (MST) of 136 cases treated with complete resection was 66 months, whereas that of 43 cases involving unresectable but limited tumors was 19 months $(p<0.0001)$ [2]. Sasaki et al. [3] reported that the MST was only 8 months in 30 scalp or face angiosarcomas, most of which were limited tumors and were treated primarily with radiotherapy (2 cases were treated with surgery and radiotherapy) at the median prescribed dose of $68 \mathrm{~Gy}$. However, only 6 patients survived more than 2 years, of which 4 patients were treated primarily with radiotherapy of more than 60 Gy and 2 patients were treated with surgery and adjuvant radiotherapy. No patients treated with radiotherapy of less than 60 Gy survived more than 2 years. Only a few of the patients with angiosarcoma who receive radiotherapy given at higher doses have a chance of long-term disease-free survival and are possibly cured. The rIL-2 was administered in combination with chemotherapy and radiotherapy in case 1 . There is a report that high total dose administration of rIL-2 $\left(>1100 \times 10^{4}\right.$ units) improved the distant metastasis-free rate significantly [4]. In our case, the high total dose of rIL$2\left(2065 \times 10^{4}\right.$ units $)$ was administered to prevent distant metastases. The significance of rIL-2 is still unclear. Chemotherapy is used alone for metastatic angiosarcoma or as an adjuvant therapy for eliminating micrometastases after the primary localized treatment. PTX has recently been used for metastatic angiosarcoma. The ANGIOTAX study is a phase II trial which investigates the efficacy and toxicity of weekly PTX for patients with metastatic or unresectable angiosarcoma [4]. PTX was administered intravenously at a dose of $80 \mathrm{mg} / \mathrm{m}^{2}$ on days 1,8 , and 15 of a 4 -week cycle in 30 patients. Toxicity was tolerable except for one grade 5 adverse event owing to thrombocytopenia in a context of general condition deterioration.
There are few reports about the concurrent use of chemotherapy and radiotherapy in the treatment of angiosarcoma. However, PTX has been safely used in combination with radiotherapy to treat other tumors, such as lung cancer. Several studies reported the radiosensitization of PTX $[5,6]$. So we treated radiation-induced angiosarcoma with concurrent PTX and radiotherapy. The dose of PTX was reduced from 80 to $70 \mathrm{mg} / \mathrm{m}^{2}$ because of the fear of increased toxicity with the use of concurrent chemoradiotherapy.

Previous authors have suggested the following criteria for diagnosing radiotherapy-associated sarcoma [7]:

1. There must be at least 3-5 years between the radiotherapy and sarcoma development.

2. The site of origin must be within the radiotherapy field.

3. The induced sarcoma must be histologically different from the primary cancer.

Case 1 involved an angiosarcoma from the right groin to the lower abdomen, which occurred about 8 years after whole pelvic radiotherapy for carcinoma of the endometrium. Case 2 involved an angiosarcoma in the right groin, which occurred about 13 years after whole pelvic radiotherapy for carcinoma of the cervix. Both cases met all the criteria.

Surgery is generally performed in radiation-induced angiosarcoma and radiotherapy is usually avoided. There are no prospective studies and some reports about the treatment of radiation-induced angiosarcoma by radiotherapy. We treated radiation-induced angiosarcoma with concurrent PTX and radiotherapy because the tumors in our patients were inoperable. As far as we know, this is the first report to describe the concurrent use of radiotherapy and weekly PTX to treat radiation-induced angiosarcoma. Our patients already received irradiation with a total dose of 45-50.4 Gy, so we treated radiation-induced angiosarcoma with a total dose of $50 \mathrm{~Gy}$, which was not enough to cure angiosarcoma, because severe toxicity of skin and subcutaneous tissue was feared. The initial responses of both cases in this report were fairly good, although local recurrence occurred in both cases a few months after the end of the chemoradiotherapy. This might have been due to the large tumors and low radiation doses involved. Some reports have stated that tumor size was a prognostic factor $[2,3]$, and both tumors described in this report were large.

The patients' initial responses to the treatment were good, and their quality of life was improved. However, the long-term control of radiation-induced angiosarcoma is difficult, probably because of reirradiation with lower doses. It is plausible that the use of concurrent chemoradiotherapy is one of the safe and effective palliative treatments, because chemoradiotherapy improves the patients' quality of life without severe acute adverse events. 
Conflict of interest The authors declare that they have no conflict of interest.

\section{References}

1. Young RJ, Brown NJ, Reed MW et al (2010) Angiosarcoma. Lancet Oncol 11:983-991

2. Lahat G, Dhuka AR, Hallevi H et al (2010) Angiosarcoma. Ann Surg 251:1098-1106

3. Sasaki R, Soejima T, Kishi K et al (2002) Angiosarcoma treated with radiotherapy: impact of tumor type and size on outcome. Int $\mathrm{J}$ Radiol Oncol Biol Phys 52:1032-1040
4. Penel N, Bui BN, Bay JO et al (2008) Phase II trial of weekly paclitaxel for unresectable angiosarcoma: the ANGIOTAX study. J Clin Oncol 26:5269-5274

5. Chen Y, Pandya K, Keng PP et al (2001) Schedule-dependent pulsed paclitaxel radiosensitization for thoracic malignancy. Am J Clin Oncol 24:432-437

6. Hiro J, Inoue Y, Tomiyama Y et al (2010) Possibility of paclitaxel as an alternative radiosensitizer to 5-fluorouracil for colon cancer. Oncol Rep 24:1029-1034

7. Kiyohara T, Kumakiri M, Kobayashi H et al (2002) Spindle cell angiosarcoma following irradiation therapy for cervical carcinoma. J Cutan Pathol 29:96-100 